Review

\title{
MUCOPOLYSACHARIDOSIS II AND SURGERY /REVIEW/
}

\author{
K. Kalinova ${ }^{1}$, K. Georgiev ${ }^{2}$, I. Mladenova ${ }^{2}$ \\ ${ }^{1}$ Department Pediatric Surgery, Medical Faculty, Trakia University, Stara Zagora, Bulgaria \\ ${ }^{2}$ Department of Epidemiology, Medical Faculty, Trakia University, Stara Zagora, Bulgaria
}

\begin{abstract}
Patients with type mucopolysaccharidosis/MPS/ II usually undergo surgery at an early age before the diagnosed. Mucopolysaccharidosis, type II is also known as Hunter syndrome.Recurrent early surgical interventions, especially for hernia or carpal tunnel syndrome, are characteristic of these patients.
\end{abstract}

Key words: mucopolysacharosis, children, surgery

\section{INTRODUCTION}

Mucopolysaccharidosis, type II is inherited Xlinked and is due to deficiency of the enuronate sulfate enzyme, which leads to the accumulation of dermate sulfate and heparan sulfate. Glucosaminoglycans are accumulated in the lysosomes and excreted in the urine. Dermatan sulphate, heparan sulphate, keratan sulphate, chondroitin sulphate are the main glucoseaminoglycans in the tissues. Depending on the enzyme deficiency, the catabolism of one or more glucoseaminoglycans may be impaired $(1,2)$.

Heparan sulfate is an important component of nerve cell membranes, and its accumulation leads to mental disorders. The accumulation of keratin sulfate causes skeletal deformities. Dermatan sulfate is found mainly in the skin, but also in the blood vessels, heart valves, lungs, tendons.

Hunter syndrome differs from other mucopolysaccharidoses in that it is an X-linked disease.

Two forms of the syndrome are known - severe (type A) and mild (type B). In its most severe form, clinical manifestations are manifested

Correspondence to: Krasimira Kalinova MD, PhD, Department of Pediatric surgery, Medical Faculty, Trakia University, Armeiska 18-A-19, 6003 Stara Zagora, Bulgaria, Tel. 359 889424292, E-mail: krasimirakalinova@abv.bg during the first few years of life and end fatal by the time of adolescence. The reason is usually the development of heart and respiratory failure.

Type A has clinical symptoms similar to Hurler syndrome except corneal blurring. In addition, the progression of symptoms is not as rapid as in Hurler syndrome. Development is delayed. Children are often deaf and survive until the second - third decade of life.

Children with type B mucopolysaccharidosis type II resemble those with Hurler - Scheie syndrome or with Scheie syndrome. They have normal intellectual development but develop airway obstruction due to the accumulation of mucopolysaccharides in the trachea and bronchi. Most patients develop heart valve disease, but they survive to the seventh decade of life.

The clinical picture of type II mucopolysaccharidosis is very specific. The disease is found with increased frequency among the Jewish population in Israel. The severe form of Hunter syndrome is diagnosed in children 2-4 years old. The mild form is detected during adolescence or later (1).

The severe form (type A) is characterized by a progressive involvement of the nervous system. Typical symptoms are rough facies, low stature, skeletal deformities, joint stiffness, and mental retardation. Hyperactivity, progressive hearing loss, hepatomegaly, carpal tunnel syndrome, retinal degeneration, and 
recurrent ear infections may also be observed. The involvement of the gastrointestinal tract leads to the development of chronic diarrhea or constipation.

Normal intelligence is observed in type B, although other signs are less pronounced. Death occurs as a result of an obstructive or cardiac disorder usually after the fifth decade of life (1).

The diagnosis of mucopolysaccharidosis, type II, is of particular importance for specialized studies and the clinical picture.GAGs are accumulate in the lysosomal deficiency of 11 different enzymes causes different forms of MHC (2).

MPC I reduces the activity of alpha-L iduronidase, which is involved in the breakdown of dermatan and heparan sulfate. It is inherited autosomal recessively with over 100 mutations in the IDUA described so far. The frequency of this form is about 1 in $100,000(3,4)$.

Traditionally, MHC I is subdivided into three subtypes, depending on the severity clinical picture:

- Hurler's disease (MH IH, severe);

- Hurler-Scheie disease (MH IH/S, moderate);
- Scheie's disease (ISD, mild).

The three subtypes are biochemically indistinguishable and are characterized by progressive multisystem involvement.

MHC I represents a wide range of overlapping clinical phenotypes, so, from a practical point of view, it is more convenient to subdivide it into severe (MHC IH) with progressive CNS involvement and an attenuated form (MHC IH / $\mathrm{S}$ and IS) with different severity of visceral symptoms, but without neurological damage $(5,6)$.

All types of MPS disorders are rare, including Hunter syndrome, which affects primarily males, approximately 1,500 to 2,000 patients worldwide. This occurs in at least 1 in 162,000 and up to 1 in 34,000 live male births, depending on the population studied.

- Bulgaria is the first country in Eastern Europe to adopt a national program for rare diseases in November 2006. The national program 2009-2013 was prepared in accordance with the European and national Health strategies (Table 1).

- 13 centers of expertise in Bulgaria. Seven are members of a European reference network

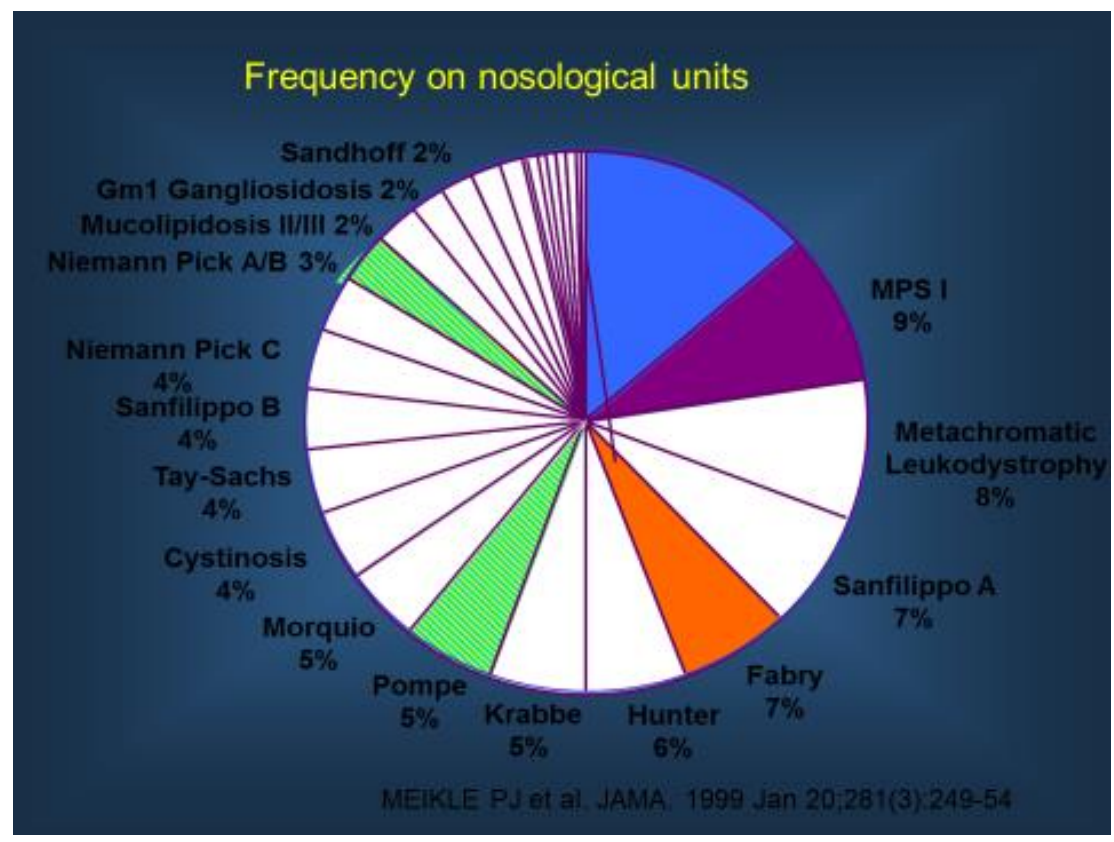

Figure 1. Frequency on nosological units (7)

\section{Etiology and pathogenesis}

People affected by Hunter syndrome are unable to break down two specific GAGs: dermatous sulfate (found in the skin, blood vessels and heart) and heparan sulfate (found in the lungs, arteries, and cell surface). This is due to the fact that they lack an enzyme called induronate-2-sulfatase.There are 7 different 
types of vehicles, the subject of our presentation is 2 special types. Each MPS type is characterized by progressive craniofacial, joint and skeletal deformities, cardiac involvement and early death due to pulmonary infections or heart failure, often before maturity. In the MPSII have ENT problems and can be observed in all patients the problems in peripheral nervous, gastrointestinal and dermatological systems (1).

Table 1. Perspectives of the Republic of Bulgaria 1980-2017

\begin{tabular}{|c|c|c|c|}
\hline \multirow{2}{*}{ Patients } & Enzime & ДNA & Prenatal \\
\hline & $\underline{\text { Diagnostic }}$ & $\underline{\text { Diagnostic }}$ & $\underline{\text { Diagnostic }}$ \\
\hline & & & 12 \\
\hline 26 Buloaria & 21 & 15 & 4 \\
\hline 204 Macedonia & 3 Munster & 12 Bulgaria & Rotterdam \\
\hline 2 Serbia & (Before 2000) & 3 Macedonia & $\begin{array}{l}\text { (Before } \\
2012 \text { ) }\end{array}$ \\
\hline
\end{tabular}

Hunter syndrome is categorized as, genetic disorder with:

- two copies of the X chromosome
- Normally have two copies of the I2S gen Males /Have one X and a Y chromosome

- Have only on copy of the I2S gene X chromosome)

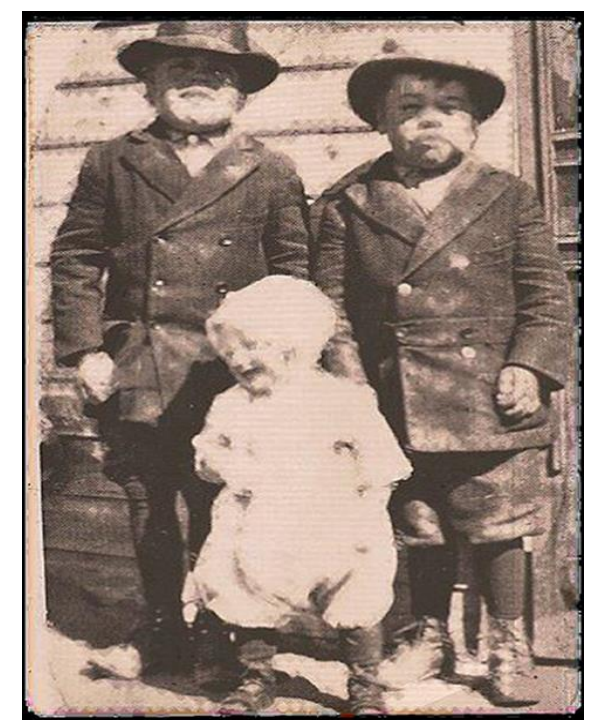

Figure 2. Hunter syndrome in Winnipeg General Hospital /1917/

In 1917 Dr. Charles Hunter-Canada describe for two brothers /aged 10 and 8 years in Winnipeg General Hospital with the general for this disease symptoms: low height, macrocephalus, deafness, cardiomegaly, umbilical hernia, joint contracts, bone involvement $(3,4)$. (Figure 2)

\section{Clinical picture}

Because dermatan and heparan do not degrade, their levels increase, affecting the way some cells and organs function in the body. This leads to several serious symptoms. The physical features of Hunter syndrome earing and breathing problems. They can develop heart problems or stiffcan vary greatly.

Umbilical or inguinal hernias (small lump or bulge around navel or groin area) $25-30 \%$ in newborns (in the case of premature babies: 70 $75 \%)$ Figures 3a and b (5). 


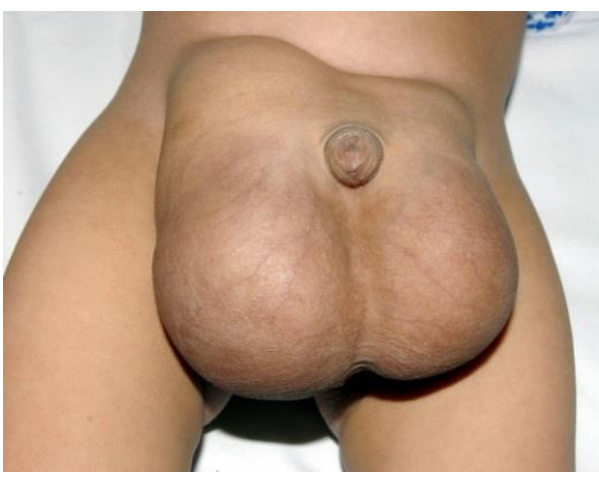

Figure 3a. Hunter syndrome with bilateral inguinal hernias

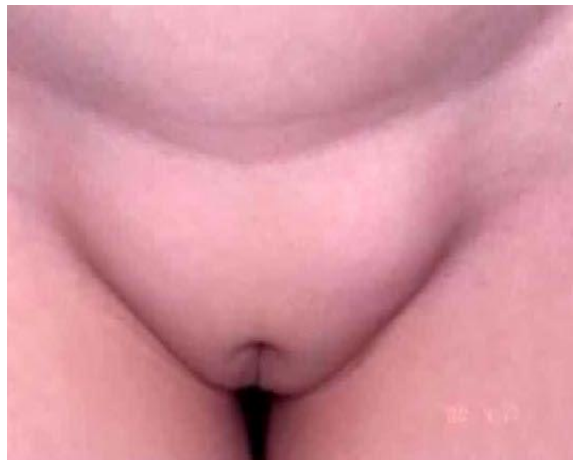

Figure 3b. Hunter syndrome in girl with inguinal hernias
KALINOVA K., et al.

- $5 \%$ for children up to 3 years of age

- $2-4 \%$ in children (preterm: 9-11\%)

- 2 - 4 times more often to the right

- $10-20 \%$ bilateral

The most common surgical intervention in childhood.

Umbilical hernia tends to spontaneous resolution. It is recommended to have surgery after 2 years of age

- Ear infections

- Running nose

- Colds

- Because these symptoms are quite common in all newborns, it is unlikely that they will persuade the doctor to diagnose or even immediately think about Hunter syndrome. Sleep breathing problems, including respiratory arrest as well as noisy daytime breathing - Limited lung capacity - Joihis can lead to stiffness when moving or walking $\bullet$ Carpal tunnel syndrome $\cdot$ Rough, ivoryskin lesions on upper arms and legs $\bullet$ Developmental disability - Poor nutrition and diarrhea

- $15 \%$ for children under 1 year of age
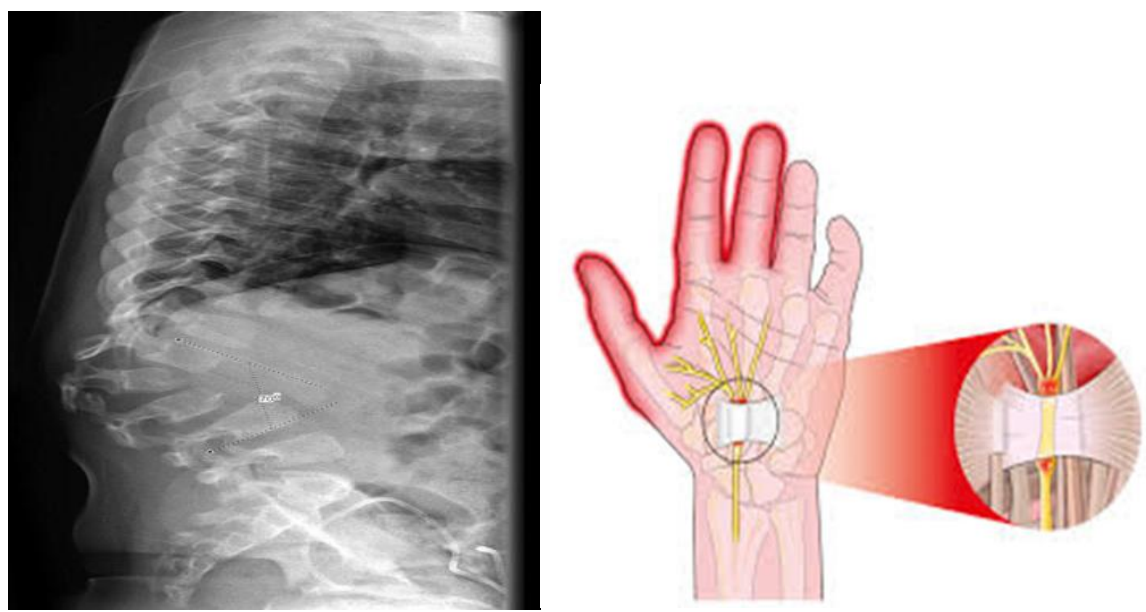

Figure 4. Joint-bone changes in MPS II $[4,6]$.

- Limited movement in the joints, shortened muscles and raised muscular tonus

- Musculo-articular contractions

- bone-joint deformities

- thickening / flattening of bones and joints,kyphosis,scoliosis

- short neck,pectus excavatum

- Dog cavus

- Strong and progressive shortening of the Achilles tendon

- Abdominal wall protrusion (hepatomegaly)
- Progressive arthropathies affecting all joints

- Dysplasia of TBC

- Claw-hand deformity /Fig.4/

\section{Diagnosis of Hunter syndrome}

When diagnosed with mucopolysaccharidosis, they are based on complaints, anamnestic data, the results of additional testing methods physical, instrumental, and laboratory. One can suspect the presence of mucopolysaccharidosis from the characteristic deformities of the 
skeleton that "catch the eye", but it is possible to determine the type of the disease described only by using additional methods of investigation. The main methods are: X-ray; detection of glycosaminoglycans in urine; determination of enzyme activity in cell cultures.

- Urine testing screens for presence of GAG; increased levels are suggestive of MPS, but not definitive for Hunter syndrome

- I2S protein concentration and enzyme activity to confirm suggestive diagnosis and results of urine GAG test

- Genetic testing identifies presence of defective I2S gene, making definitive diagnosis of Hunter syndrome. Genetic testing recommendations.

- Prenatal testing via amniocentesis in mother of an affected child or first-degree female relative of a carrier or affected child

- Skeletal abnormalities are early and prominent features of MPS, and the orthopedic surgeon is often the first healthcare provider to raise suspicion [3].

- The orthopaedic surgeon should have a basic understanding of MPS and of the clinical presentation, musculoskeletal abnormalities, and radiographic findings. $(4,6,7)$.

- Treatment of skeletal manifestations usually involves surgical intervention.

- Regular imaging of the cervical, thoracic and lumbar spine, the hips and the lower extremities is recommended for the treatment of diseases of the skeletal system

- The flexibility of the joints of the child can be improved with physiotherapy that overcomes stiffness and maintains function. However, physical therapy cannot stop the gradual reduction of joint mobility. The child may eventually need a wheel chair due to pain and restricted movement

- Not only known stigmas but also concomitant surgical pathology signals the presumed diagnosis of MPS. One of most common nosological units is umbilical and inguinal hernia. They are subject to surgical treatment, but due to the weakness of the connective tissue, the operative result is not always positive. Repeated operations are often required. In this regard, some recommend wearing bandages to avoid surgery.

- Patients with Hunter syndrome have perioperative complications in about $50 \%$. In this syndrome, the incidence of
KALINOVA K., et al. respiratory tract problems such as respiratory tract narrowing, laryngomalacia, profuse secretions and pulmonary dysfunction is $53 \%$. $(8,9)$.

\section{Management of symptoms and complications associated with Hunter syndrome}

According to the study, children with MDH had their first surgery at an average age of 2.6 years. For example, tympanostomies and herniotomy for inguinal and umbilical hernias well as those are the most common [10]. Recently developed medical therapies for the management of MPS (hematopoietic stem cell transplantation, intravenous enzyme replacement therapy) have led to increased lifespan but have not had much effect on the development of skeletal deformities. Patients must be monitored carefully and treated surgically as necessary. Conditions that may require surgical management include cervical spine and atlantoaxial instability, gibbus deformity, hip dysplasia and osteonecrosis, genu valgum, and carpal tunnel syndrome.

Breathing and airway problems: administration of oxygen, use of breathing devices such as CPAP and BiPAP or endotracheal tubes, surgery to remove adenoids or tonsils, and asthma medications. Infections: long-term use of antibiotics, prophylactic antibiotics before dental procedures may be needed Ear infections and hearing problems: tympanostomy tubes, hearing aids. Cardiac complications: antihypertensive for blood pressure control hypertension, heart valve replacement for valvular disease (10).

The severity of the disease depends on the residual activity of enzymes. Life expectancy is limited to ten or twelve years in severely affected patients, while those with a milder disease can reach adulthood. A sign of the alleged diagnosis of MPS is not only the known stigmas but also the accompanying surgical pathology (11). One of the most common nosological units is the umbilical and inguinal hernia (12). They are subject to surgical treatment, but due to the weakness of the connective tissue, the operating result is not always positive. Repeat operations are often required. The treatment of MPS included: Symptomatic/ maintenance treatment; Transplantation of hematopoietic stem cells, Enzyme replacement and gene therapy (13). In this regard, some recommend the wearing of bandages to avoid surgery $(14,15)$. 
- Adenoidectomy, tonsillectomy Hernia repair (umbilical and inguinal)

- Carpal tunnel release Tracheostomy, Ttubes, Portacath, Hernia repair (umbilical and inguinal)

- Carpal tunnel release Hip replacement

- Dental surgery Appendectomy

- Mastoidectomy

Torsion of the testis

- Heart valve replacement $\backslash$ Hip replacement

\section{CONCLUSIONS}

Raising awareness and knowledge about mucopolysaccharidoses among pediatricians and general practitioners is of utmost importance for early diagnosis and treatment, especially considering treatment outcomes which are optimal if treatment is started prior irreversible changes (damage) occurs.

Patients with type II mucopolysaccharidosis usually undergo surgery at an early age, often before the MPS is diagnosed. Recurrent early surgical interventions, especially for hernia or carpal tunnel syndrome, are characteristic of these patients. The suspicion of a MHC should be a signal for a careful approach to anesthesia and to monitor for possible complications in the perioperative period. Early diagnosis of MPS II is important for optimizing the treatment of the disease.

Acknowledgement for Dr. N. Malechkova, Shier

\section{REFFERENCES}

1. Aronovich EL, Pan D, Whitley CB. Molecular genetic defect underlying alpha L-iduronidase pseudodeficiency.Am J Hum Genet.; 58 (1):75-85, 1996

2. Clarke L, Wraith JE, Beck M, Kolodny EH, Long-term Efficacy and Safety of Laronidase in the Treatment of Mucopolysaccharidosis $\quad$ I. Pediatrics.123: 229-240,2009

3. dDeRu MH, Boelens JJ, Das AM et al. Enzyme replacement therapy and/or hematopoietic stem cell transplantation at diagnosis in patients with mucopolysaccharidosis type I: results of a European consensus procedure. Orphanet $J$ Rare Dis. 6:55-57. 2011

4. Muenzer,Wraith JE, Clarke LA, MucopolysaccharidosisI:Management and Treatment Guidelines. Pediatrics.123:19_ 29. 2009
KALINOVA K., et al.

5. Brankov O. Pediatric surgery,Marin Drinov,Sofia,Bulgaria 2011

6. Muhlebach MS, Wooten W., Muenzer J.,Respiratory manifestations in mucopolysaccharidoses.

Paediatric Respiratory Reviews, 12:133-138. 2011

7. Souillet G, Guffon N, Maire I, et al. Outcome of 27 patients with Hurler's syndrome transplanted from either related or unrelated haematopoietic stem cell sources.

Bone

Marrow Transplant. 31:1105-1117, 2003

8. Taylor C, Brady P, O'Meara A, et al. Mobility in Hurler syndrome. $J$ Pediatr Orthop. 28:163-8, 2008

9. Nancy J. Mendelsohn,a.al.Importance of surgical history in diagnosing mucopolysaccharidosis type II (Hunter syndrome): Data from the Hunter Outcome Survey Genetics IN Medicine, 12: 81682,2010

10.Terlato NJ, Cox GF: Can mucopolysaccharidosis type I disease severity be predicted on a patient's genotype?A comprehensive review of the literature. Genet Med,5:286-94,2003

11.Vijay S, Wraith JE. Clinical presentation and follow-up of patients with the attenuated phenotype of mucopolysaccharidosis type I. Acta Paediatr.; 94:872-72005.

12.Walker RW, Darowski M, Morris $\mathrm{P}$ et al.Anaesthesia and mucopolysaccharidoses.A review of airway problems in children. Anaesthesia.49:107884,1994

13.Murray RK, Keeley FW. Chapter 48: The Extracellular Matrix in Harper's Illustrated Biochemistry, 29th Edition. McGraw- Hill Education, New York, 2012.

14.Rest Technologies.CPAP Illustration. Available at http://www.rest-technologies. com/images/cpap_treatment.jpg. Accessed December 1, 2010.

15.15.Nancy J Mendelsohn a.al. Importance of surgical history in diagnosing mucopolysaccharidosis type II (Hunter syndrome): Data from the Hunter Outcome Survey.Genetics in Medicine.12,816-822, 2010 